\title{
Pediatric Mastocytosis: A Review of the Literature
}

\author{
Marianne Frieri, MD, PhD, and Mahvish Quershi, BA
}

Mastocytosis has a bimodal distribution often presenting in children from birth to 2 years of age and in those over the age of 15. Pediatric mastocytosis is due to the effects of mast-cell degranulation enzymes such as histamine and tryptase causing the presentation of pruritis, flushing, vesicles, abdominal and bone pain, or headache. Three different forms of mastocytosis can occur: urticaria pigmentosa, diffuse cutaneous, and solitary mastocytoma. Systemic symptoms are typically a result of mast-cell mediator release but do not prove systemic mast-cell hyperplasia. In this review, we present several research studies related to pediatric mast-cell disorders, and discuss several cases of pediatric mastocytosis, acute myeloid leukemia, pathophysiology, genetic studies, and treatment.

\section{Introduction and Research Studies}

$\mathrm{P}$ EDIATRIC MASTOCYTOSIS CAN very easily be mistaken for a variety of common rashes that plague the pediatric population. Children very commonly present to pediatricians with different rashes ranging from urticaria to eczema to poison ivy. Pediatric mastocytosis is a rare disease, which affects the skin and multiple organs due to an increase in mast-cell load in the body. Mast cells participate in immune defense and allergic disease. When mast cells degranulate, two of the main enzymes released are histamine and tryptase, which participate in the skin reaction that appears as a rash and is classified as mastocytosis. Mastocytosis has a bimodal distribution, with $55 \%$ of cases presenting from birth to 2 years of age, $10 \%$ in children younger than 15 years old, and $35 \%$ in those over the age of 15 . There has, however, been no gender bias noted in the cases of pediatric mastocytosis. ${ }^{1}$

Pediatric mastocytosis is generally a benign disease that is transient in nature, as there is generally a spontaneous regression of the condition by puberty. Clinical presentation was evaluated in an earlier study conducted by the National Institutes of Health (NIH) in which $83 \%$ of the children presented with pruritis, $65 \%$ with flushing, 53\% with vesicles, $41 \%$ with abdominal pain, $18 \%$ with bone pain, and least commonly $12 \%$ with headache. ${ }^{1}$ Due to the high levels of histamine being released from the mast cells, there is also an increase in gastric-acid secretions resulting in gastrointestinal bleeding and peptic ulcers in the mastocytosis patient population. ${ }^{1}$

The most common form of mastocytosis in the pediatric population is cutaneous mastocytosis, in which the mast-cell hyperplasia occurs in the skin. The three major types of $\mathrm{cu}-$ taneous mastocytosis are urticaria pigmentosa (UP), diffuse cutaneous mastocytosis (DCM), and solitary mastocytoma. Mast cells degranulate in various organ systems other than the skin, and this is classified as systemic mastocytosis.

\section{Mast-Cell Hyperplasia}

In order to analyze the extent of mast-cell hyperplasia, core bone-marrow biopsies were performed and analyzed. In one study, it was found that in 10 of 17 children with mastocytosis, there were focal areas of mast-cell hyperplasia, which had perivascular and paratrabecular aggregates of mast cells, eosinophils, and early myeloid cells. ${ }^{1}$ It was also very importantly noted that mast-cell hyperplasia is not pathognomonic for mastocytosis, as mast-cell hyperplasia was also noted in 3 out of 16 control pediatric patients with other hematologic diseases. ${ }^{1}$

Another study found that the bone-marrow lesions of children had small, mostly perivascular confluent clusters of mast cells with round and oval nuclei rather than spindleshaped mast cells, as found in adult bone-marrow lesions. ${ }^{2}$ It was noted that the confluent clumps of mast cells were not found in smears of pediatric cases of marrow mast-cell hyperplasia due to non-mast-cell disorders, such as Ewing's Sarcoma, Wiskott-Aldrich syndrome, and so on. ${ }^{2}$ In comparison, in bone-marrow biopsies of adults with mastocytosis, there were fusiform mast cells that were spindle shaped, with lymphocytes and eosinophils, found in perivascular, peritrabecular, and intratrabecular locations. ${ }^{1,2}$ Overall when quantifying mast-cell hyperplasia, there was a higher load of mast cells in children with mastocytosis than

Department of Allergy and Immunology, Nassau University Medical Center, East Meadow, New York. 
in adults with mastocytosis. ${ }^{3}$ One study found a different histopathology of pediatric mastocytosis, with more extensive mast-cell infiltrates not only around blood vessels but throughout the dermis and disrupting normal skin architecture. ${ }^{3}$ Based on these findings, the difference in presentation between adult and pediatric cases of mastocytosis may result from the difference not only in the mast-cell load but also the distribution of where the mast cells are most likely to be found.

\section{Role of Histamine}

Histamine is the major enzyme released during mast-cell degranulation. Normal plasma levels of histamine range from about 0.3 to $1.0 \mathrm{ng} / \mathrm{mL}$. It was found that histamine levels were increased in DCM, and were seven times the normal amount in UP. ${ }^{1}$ In another study, it was found that in patients with UP, there was an average of about $0.9 \mathrm{ng} / \mathrm{mL}$ plasma histamine level, and in patients with DCM the plasma histamine level was about $158 \mathrm{ng} / \mathrm{mL}^{3}$ This increase in histamine levels was not found to correlate with the mastcell load in the skin lesions. In the patient with UP, it was found that there was about 3,200 mast cells $/ \mathrm{mm}^{2}$ by biopsy, and in DCM there were about 2,700 mast cells $/ \mathrm{mm}^{2}$. While there is not a correlation between the number of mast cells in the affected lesions and the plasma histamine concentrations, there may be an increase in plasma histamine levels based on the total body mast-cell burden when taking all lesions into consideration. ${ }^{3}$ On the other hand, a correlation was found between an increase in plasma and urine histamine levels and the extent of bone involvement and basal gastric acid output in mastocytosis patients. ${ }^{3}$

\section{Research Studies Related to Histamine and Tryptase}

Tryptase is the other main enzyme that is released from mast cells. Normally the levels in the body are $<11.5 \mathrm{ng} /$ mL. ${ }^{4}$ Tryptase levels can be used to reflect the mast-cell burden in the body. Similar to histamine-level findings, it was found that tryptase levels in patients with DCM were higher than tryptase levels found in patients with UP. ${ }^{4}$ It was also noted that tryptase levels have a pattern of evolution in children. The total tryptase levels are highest in young infants at about $6.12 \pm 3.47 \mu \mathrm{g} / \mathrm{L}$, and the levels decreased with age to $3.85 \pm 1.8 \mu \mathrm{g} / \mathrm{L}$ by the time the infants reached $9-12$ months of age. ${ }^{4}$ Tryptase levels were analyzed in 72 atopic and allergic infants under the age of 3 months; in 242 nonatopic, nonallergic infants who were getting a routine blood draw; and in 58 nonatopic nonallergic infants under the age of 3 months who required intensive care. It was found that the tryptase levels in the 72 atopic and allergic infants was highest at $14.20 \pm 10.22 \mu \mathrm{g} / \mathrm{L}$, and the 58 nonatopic, nonallergic infants requiring intensive care had the lowest tryptase levels at $4.12 \pm 3.38 \mu \mathrm{g} / \mathrm{L}^{4}$

Tryptase levels were also found to correlate with the scoring index of mastocytosis (SCORMA) ratings in mastocytosis patients. ${ }^{5}$ SCORMA is based on a semi-quantitative analysis of the extent, intensity, and subjective complaints, and each item is scored from 0 to 3 , where 0 is for an absent item and 3 represents the highest severity. ${ }^{6}$ Five subjective symptoms-provocative factors, flushing, diarrhea, pruritus, and local bone pain-are scored by patients from 0 to 10 using a visual analogue scale. The formula $\mathrm{A} / 5+5 \mathrm{~B}+2 \mathrm{C} / 5$ is used to calculate the final SCORMA score. The value of the SCORMA score ranges from 5.2 to 100 . This method provides standardized information on the severity of cutaneous mastocytosis and imposes no burden on the patient, which is particularly important in children. ${ }^{6}$ In patients with DCM, a tryptase level of $49.70 \pm 42.64 \mu \mathrm{g} / \mathrm{L}$ was found with a corresponding high SCORMA level of $82.57 \pm 4.06$. In patients with UP, a tryptase concentration of $7.94 \pm 15.62 \mu \mathrm{g} / \mathrm{L}$ was found with a corresponding SCORMA rating of $41.61 \pm 12.46$. Patients with mastocytoma had a tryptase level of $4.83 \pm 3.39$ $\mathrm{ug} / \mathrm{L}$ with a SCORMA rating of $28.5 \pm 9.23 .^{4}$ With this correlation between SCORMA and tryptase levels in patients with mastocytoma, it has been found that tryptase can be used for diagnostic purposes as well as a marker for following the course of mastocytosis in a patient.

Serum baseline total tryptase levels in 111 children with cutaneous mastocytosis, 80 maculopapular cutaneous mastocytosis/plaque mastocytosis, 22 nodular mastocytosis, and 9 DCM were investigated by Alvarez-Twose as a predictive biomarker for the occurrence of mast-cell mediator-related signs and symptoms within the first 18 months after the onset of disease. ${ }^{7}$

Twelve children (11\%) who showed extensive cutaneous disease involving $>90 \%$ of body surface area suffered from severe symptoms requiring hospitalization, with or without management in the intensive care unit because of lifethreatening complications that included upper gastrointestinal bleeding in the absence of coagulopathy, sepsis secondary to severe cutaneous infections caused by Enterobacter cloacae, Staphylococcus epidermidis and Staphylococcus aureus, and/or hypotension requiring epinephrine, massive mast-cell mediator release triggered by heat, rubbing, and fever in all cases. Sustained hypertension alternating with hypotensive episodes was observed in one patient from extensive maculopapular cutaneous mastocytosis who underwent chronic antihypertensive drug therapy, but with periods when therapy was withheld because of hypotensive episodes requiring treatment with epinephrine. ${ }^{7}$

The median baseline total tryptase was significantly $(p<0.001)$ higher in patients with extensive cutaneous disease compared with those with $<90 \%$ of their body surface area involved, as well as in children with grade 4 (severe mastocytosis-related symptoms requiring emergency therapy and hospitalization) compared with those with grade $<4$.

Receiver-operating characteristics curve analyses showed that the optimal cutoffs for serum baseline total tryptase to predict the need for daily antimediator therapy, hospitalization, and the management in an intensive care unit were $6.6,15.5$, and $30.8 \mu \mathrm{g} / \mathrm{L}$ respectively. Thus, increased serum baseline total tryptase in association with extensive cutaneous involvement identifies patients at risk for severe mastcell activation events in pediatric mastocytosis. ${ }^{7}$

Carter examined perianesthetic records of patients with pediatric mastocytosis who were anesthetized for diagnostic and surgical procedures from 1993 to 2006, and conducted a literature review of the experience of the use of anesthetics for pediatric mastocytosis. ${ }^{8}$ Twenty-two patients with pediatric mastocytosis, with a median age of 3.2 years (range 6 months to 20 years) at the time of the procedure, were anesthetized for 29 diagnostic and surgical procedures. All variants of the disease are represented in this series. Most patients had a history of flushing, pruritus, 
gastroesophageal refux disease (GERD), and abdominal pain; one patient had history of spontaneous anaphylaxis. Routine anesthetic techniques were used, and despite the complexity of the disease, the perioperative courses were uncomplicated and without serious adverse events. The authors reviewed the main features of pediatric mastocytosis and its anesthetic and perioperative implications, and described a practical approach to the anesthetic management of pediatric patients. The authors felt while many drugs used routinely in anesthesia reportedly cause mastcell degranulation, deviations from routine anesthesia techniques are not necessarily warranted. However, an understanding of the anesthetic implications of the disease and meticulous preparation to treat possible adverse events are advised. ${ }^{8}$

\section{KIT and D816V Point Mutation Research}

Adult mastocytosis is an aggressive disease, unlike its pediatric counterpart, which is transient and relatively benign. To understand the difference in presentation, the genetic components of the disease have been analyzed. Mast cells derive from $\mathrm{CD} 34+$ bone-marrow progenitors and circulate as precursors in the peripheral blood until reaching organs where the progenitors then differentiate under the influence of growth factors such as stem cell factor (SCF) and its c-KIT receptor and nerve growth factor. Kambe reviewed KIT that encodes a transmembrane tyrosine-kinase type III receptor, and, with $\mathrm{SCF}$, it is responsible for not only mastcell growth but also for melanogenesis, normal hematopoeisis, gametogenesis, and regulation of gastric slow waves. ${ }^{9}$ It was found that in c-KIT deficient mice, there was macroanemia, loss of hair pigment, sterility, and decrease in the gastro-intestinal (GI) pacemaker cells, as well as a lack of mast cells. ${ }^{9}$ The study continued to analyze humans with KIT loss of function mutations and found piebaldism, a disorder of melanocyte development. In patients with KIT gain of function mutations, patients presented with GI tumors. $^{9}$

Earlier studies by Verzijl found that $25 \%$ of pediatric patients with UP had an activating D816V mutation present. ${ }^{10}$ Another Longley study found that $36 \%$ of pediatric patients had another mutation at codon $816 .{ }^{11}$ This mutation leads to constitutive activation of the tyrosine kinase domain resulting in consistent cell proliferation. One study found that $25 \%$ of pediatric patients with the UP form of mastocytosis had a D816V mutation. ${ }^{11}$ It was also found that 10 out of 12 patients had another missense mutation at the location. ${ }^{11}$

A later study by Bodemer in 50 pediatric patients with ages ranging from birth to 16 years found $86 \%$ of them had a c-KIT mutation, with $36 \%$ being a D $816 \mathrm{~V}$ point mutation, 2 out of 50 cases being a D816Y mutation, and one case being a D816I mutation. There were also 29 of 50 patients with the wild-type aspartic acid at the 816 codon. ${ }^{12}$ All of the mutations were not in the c-KIT; $44 \%$ of the patients were found to have a mutation outside of c-KIT with the mutations lying in exons 8,9 , and 11 . There was also a subset of $28 \%$ of the patients with a M541L mutation at exon 10. None of the patients with a M541L mutation had the wild-type aspartic acid at codon $816 .{ }^{12}$ When the blood sample of 13 patients was analyzed, it was found that there was no c-KIT mutation, which suggests that the mutation is somatic rather than germline, meaning that this mutation is not inherited from a patient's parents. $^{12}$

Yang studied associated KIT extracellular domain mutations functional and signaling properties compared with KIT-phosphotransferase domain mutations and described that half of the mastocytosis children carry mutations in extracellular domain (ECD) using flow cytometry, immunoprecipitation, immunoblotting, a cell proliferation assay, stable KIT-expressing cell lines, RNA isolation, and reversetranscriptase PCR molecular techniques. ${ }^{13}$ These authors also found that ECD mutants, but not phosphotransferase domain (PTD), mutants, activated adenosine kinase transferase (AKT) pathway, and the ECD mutant-mediated cellular functions were AKT activity dependent. Myristoylated AKT restored impaired erythroid differentiation in PTD mutantexpressing cells. Their results implied different pathogenesis of pediatric versus adult mastocytosis, which might explain their diverse phenotypes and for the first time, different activation mechanisms of ECD versus PTD mutants at both cellular and molecular levels, which might help us to understand the heterogeneity of mastocytosis better. ${ }^{13}$

\section{Acute Myeloid Leukemia and Selected Clinical Cases}

Normally acute myeloid leukemia (AML) is highly responsive to chemotherapy. Despite the differences in adult and pediatric mastocytosis, a similar interaction was found in a 48-year-old male who presented with AML and in whom mastocytosis persisted after remission. ${ }^{14}$

Pediatric mastocytosis patients occasionally present with other hematologic diseases such as AML. Gadage presented the case of a 14 year old with systemic mastocytosis and AMLt $(8 ; 21)$, who presented with abdominal pain, low-grade fevers, weakness, and numbness of the lower legs for 2 weeks. There was no pruritis or skin lesions noted. In the peripheral blood smears, there were $66 \%$ blasts with $\mathrm{MPO}+$ stains, and the bone-marrow biopsy was hypercellular with an increase in CD34+ blasts, as well as CD2 and CD25 coexpression signifying a neoplastic disease ${ }^{15}$ It is plausible that the presentation of systemic mastocytosis was masked due to the increase in blasts and mast cells at the stroma of the bone marrow. It is also possible that the CD56 and KIT mutation associated with systemic mastocytosis adversely affected the prognosis of the chemotherapy results this patient would have otherwise had. ${ }^{15}$

A concurrent diagnosis of mastocytosis and AML results in a poor prognosis for treatment of the AML with chemotherapy, as noted in a study with a child who presented with AMLt $(8 ; 21)$ and asymptomatic systemic mastocytosis. The patient had no c-KIT D816V mutation, and underwent four cycles of chemotherapy. After completion of chemotherapy, the systemic mastocytosis persisted. The patient was found to have a relapse of AML after 9 months. When the patient went into a second remission, however, there was still a persistence of mastocytosis. ${ }^{16}$

\section{Cases Chosen for This Review}

Although this is a review of pediatric mastocytosis, there is a case of interest concerning a 56-year-old black male who presented with fatigue, weight loss, postprandial nausea, vomiting, and diarrhea. There were no skin lesions, past 
medical illnesses of significance, or previous history of cigarette or alcohol use. He denied use of medications and allergies. Serum protein electrophoresis revealed increased beta globulins and a mini-monoclonal band in gamma region. Serum immunofixation revealed a biclonal gammopathy with an IgG/kappa and IgA/lambda bands. Urine immunofixation revealed a monoclonal free kappa light chain. $\mathrm{He}$ also had elevated quantitative immunoglobulins for $\mathrm{IgG}-1870 \mathrm{mg} / \mathrm{dL}$ and $\mathrm{IgA}-2000 \mathrm{mg} / \mathrm{dL}$ with a normal IgM$147 \mathrm{mg} / \mathrm{dL}$. Two years later, he presented with ascites, pedal edema, weight loss, postprandial diarrhea, weakness, and lethargy. A CAT scan revealed abdominal lymphadenopathy, heptosplenomegaly, and liver and lymph nodes with increased mast cells. ${ }^{17} \mathrm{He}$ was diagnosed with acute nonlymphocytic leukemia. ${ }^{18}$

Pediatric mastocytosis is often mistaken for more common rashes such as in one study with a 9-month-old boy who presented with a rash that was originally mistaken for a milk allergy and then for atopic dermatitis, ${ }^{19}$ or a 10month-old boy with recurrent urticarial episodes. ${ }^{20}$ Krishnan reported a solitary mastocytoma in 14-year-old boy with urticaria and angioedema. ${ }^{21}$ Lesions were extremely pruritic and several episodes of lip swelling occurred each week that would last 24 hours and which were not associated with breathing difficulties or other symptoms. He took $25 \mathrm{mg}$ of benadryl every 4-6 hours for pruritis, and could not identify a specific pattern or trigger for urticaria and angioedema. No other systemic symptoms occurred. His past history was unremarkable. Family history was negative for autoimmune disorders and angioedema. Review of systems was normal. Physical examination was remarkable for a $4 \times 6 \mathrm{~cm}$ ovalshaped, dusky red colored, blanchable macule on his inner right thigh. Stroking did not elicit Darier's sign. Solitary mastocytomas seldom cause urticaria but should always be considered in the differential diagnosis for urticaria. Mastocytomas regress by adolescence, while a small percentage remains active beyond adolescence, raising concern for progression to systemic mastocytosis. A skin lesion suspicious for a solitary mastocytoma should prompt a diagnostic evaluation with a specialist to recognize mastocytoma early. ${ }^{21}$

An 8-month-old boy presented with a maculo-papularbullous exanthema affecting head, trunk, and extremities. ${ }^{22} \mathrm{He}$ had pruritus, and positive Darier's sign. The rash started at 5 months with constant progression. Internal and neurologic examination was unremarkable. The tentative diagnosis of cutaneous mastocytosis was confirmed histologically. Serum tryptase, serum and urine histamine levels were normal. Histological bone-marrow examination showed no pathologic $\mathrm{MC}$ infiltration; 99m-TC-MDP scintigraphy was negative. Despite conservative treatment, macular and bullous lesions gradually increased in size and number. Pruritus continuously worsened. At 23 months, serum tryptase levels started to rise but remained within normal limits. Sequence genomic DNA from a skin biopsy showed a somatic deletion of the GAC nucleotide triplet encoding aa 419, aspartic acid, in exon 8 of the KIT gene a mutation responding to imatinib therapy. Treatment with $100 \mathrm{mg}$ of imatinib daily was begun. ${ }^{22}$ Imatinib inhibits BCR-ABL tyrosine kinase. Imatinib (Gleevec, Glivec) is a synthetic tyrosine kinase inhibitor used in the treatment of chronic myeloid leukemia (CML). It is specifically designed to inhibit the breakpoint cluster region (BCR)-Abelson (ABL) fusion protein that results from the chromosomal abnormality known as the Philadelphia chromosome. ${ }^{23}$
A female aged 1 year and 2 months presented with bullous skin lesions since 4 months, with intermittent diarrhea and fever. ${ }^{24}$ She had undergone treatments for intestinal parasites and pyoderma, and had flaccid or ruptured bullae with hives located on the trunk and upper and lower limbs. Blood tests, ESR, LFTs, and bone X-rays were normal. Abdominal echography showed a normal-size liver and spleen. The skin biopsies of the abdomen, thorax, and thigh confirmed the suspicion of bullous mastocytosis, mast cells $>30$ cells per high-power field. The myelogram and bone-marrow biopsy did not show any involvement, and no mast cells were identified. Colonoscopy and intestinal biopsy revealed a mucous membrane with increased mast-cell count on the lamina propria with 15 to 20 cells per high-power field. Treatment with $\mathrm{H} 1$ and $\mathrm{H} 2$ blockers was used. Disodium cromoglycate was maintained for approximately 10 months. Treatment response was slow with no bullae or digestive symptoms in the last 6 months. The child was on hydroxyzine $(1 \mathrm{mg} / \mathrm{kg} /$ day $)$ with reassessment every 4 months. ${ }^{24}$

An Indian male infant with a diagnosis of cutaneous bullous mastocytosis was referred to our pediatric intensive care unit and transferred to the burns unit. The skin appeared similar to toxic shock syndrome. Our service was consulted. Wound care was administered. A similar case in an infant with cutaneous bullous mastocytosis had a GI bleed due to elevated plasma heparin. $\mathrm{H} 1$ and $\mathrm{H} 2$ blockers along with sodium cromolyn were given. The patient recovered and was followed up by the Allergy Immunology service until adolescence with complete resolution. ${ }^{25}$

\section{Teaching Points}

Mastocytosis can present with a wide range of cutaneous manifestations such as eczematous rash, urticaria, solitary mastocytoma, maculo-papular, bullous, or TEN like, or with no cutaneous manifestation at all.

\section{Treatment}

Treatment of mastocytosis is still based on symptomatic treatment, as most of the pediatric cases are known to resolve spontaneously by puberty. In a study at the Gdansk Mastocytosis Center for Polish patients, 2 of 101 cases were found to have complete remission by puberty, and $32 \%$ of patients had partial remission by puberty. ${ }^{26}$ One of the mainstays of treatment remains to be $\mathrm{H} 1$ and $\mathrm{H} 2$ antihistamine drugs such as hydroxyzine, which works to control the itching, as well as control gastric-acid secretion. ${ }^{1}$ Chemotherapy continues to be discouraged unless there is an associated hematologic disease. $^{26}$

A study of a 14-month-old male and a 26-month-old male by Correia found that pimecrolimus cream used twice a day with oral antihistamine use for 3 months and then reduced to once a day for 2 months was found to have a positive response in the patients. Treatment was instituted with pimecrolimus cream twice a day and oral antihistamine. An almost complete response was achieved after 4 months of therapy in both patients, with no clinical evidence of recurrence after 4 years and 2 years of follow-up. ${ }^{27}$ This cream works through inhibiting calcineurin, which results in preventing the release of inflammatory cytokines and mediators from mast cells. It is known to have a positive response in other dermatological issues involving mast cells such as 
atopic dermatitis. It was concluded that continuous use works to suppress mast-cell mediated reactions by decreasing degranulation of mast cells rather than by reducing the number of mast cells. ${ }^{27}$

There has been extensive research on how and why pediatric mastocytosis presents the way it does. While the underlying pathophysiology seems to be well understood with physicians being able to follow the histamine and tryptase levels in pediatric mastocytosis patients, it seems that the exact genetic cause underlying the disease as well as the reason for the difference in presentation between the adult and pediatric population of the disease remains elusive to the research population, as there is a wide range of opposing findings in the genetic analysis of mastocytosis. ${ }^{28}$ The heterogeneity of clinical presentation of mastocytosis is typically related to the tissue mast-cell burden, symptoms due to the release of mast-cell mediators, the type of skin lesions, the age of the patient at the onset of disease, and associated hematological disorders. Therefore, a multidisciplinary approach is recommended. ${ }^{28}$

The diagnosis is established on clinical grounds and the findings on skin biopsy. Bone-marrow studies are recommended if there is suspicion of progression of disease to an adult form, if cytoreductive therapy is contemplated, or if skin lesions remain present and/or tryptase levels remain elevated after puberty. The use of chemotherapy, including kinase inhibitors, is strongly discouraged unless severe hematologic disease is present, since malignant evolution is extremely rare. ${ }^{28}$

Systemic mastocytosis in children is extremely rare. Despite great progress in diagnosis and treatment of this disease, multidisciplinary management is recommended. Further studies of pediatric systemic mastocytosis should be conducted to investigate new therapeutic approaches. ${ }^{29}$

\section{Author Disclosure Statement}

No competing financial interests exist.

\section{References}

1. Kettelhut BV, Metcalfe DD. Mastocytosis. J Invest Dermatol 1991; 96:115S-118S.

2. Parker RI. Hematologic aspects of mastocytosis. I: Bone marrow pathology in adult and pediatric systemic mast cell disease. J Invest Dermatol 1991; 96:47S-51S.

3. Kirshenbaum AS, Kettelhut BV, Metcalfe DD, Garriga MM. Mastocytosis in infants and children: recognition of patterns of skin disease. Allergy Proc 1989; 10:17-21.

4. Belhacin W, Ibrahim Z, Grandné V, Buffat C, Robert P, Gras D, Cleach I, Bongrand P, Carayon P, Vitte J. Total serum tryptase levels are higher in young infants. Pediatr Allergy Immunol 2011; 22:600-607.

5. Heide R, van Doorn K, Mulder PG, van Toorenenbergen AW, Beishuizen A, de Groot H, Tank B, Oranje AP. Serum tryptase and SCORMA (SCORing MAstocytosis) Index as disease severity parameters in childhood and adult cutaneous mastocytosis. Clin Exp Dermatol 2009; 34:462-468.

6. Lange M, Bogusław Nedoszytko B, Górska A, Żawrocki A, Sobjanek M, Kozłowski D. Mastocytosis in children and adults: clinical disease heterogeneity. Arch Med Sci 2012; 8:533-541

7. Alvarez-Twose I Vañó-Galván S, Sánchez-Muñoz L, Morgado JM, Matito A, Torrelo A, Jaén P, Schwartz LB, Orfao A, Escribano L. Increased serum baseline tryptase levels and extensive skin involvement are predictors for the severity of mast cell activation episodes in children with mastocytosis. Allergy 2012; 67:813-821.
8. Carter MC, Uzzaman, A, Scott LM, Metcalfe DD, Quezado Z. Pediatric mastocytosis: routine anesthetic management for a complex disease. Anesth Analg 2008; 107:422-427.

9. Kambe N, Longley BJ, Miyachi Y, Kabashima K. KIT masters mast cells in kids, too. J Invest Dermatol 2010; 130:648-650.

10. Verzijl A, Heide R, Oranje AP, van Schaik RH. C-kit asp-816-val mutation analysis in patients with mastocytosis. Dermatology 2007; 214-220.

11. Longley BJ, Metcalfe DD, Tharp M, Wang X, Tyrrell L, Lu SZ, Heitjan D, Ma Y. Activating and dominant inactivating c-KIT catalytic domain mutations in distinct clinical forms of human mastocytosis. Proc Natl Acad Sci U S A 1999; 96: 1609-1614.

12. Bodemer C, Hermine O, Palmérini F, Yang Y, GrandpeixGuyodo C, Leventhal PS, Hadj-Rabia S, Nasca L, GeorginLavialle S, Cohen-Akenine A, Launay JM, Barete S, Feger F, Arock M, Catteau B, Sans B, Stalder JF, Skowron F, Thomas L, Lorette G. Plantin P, Bordigoni P, Lortholary O, de Prost $Y$, Moussy A, Sobol H, Dubreuil P. Pediatric mastocytosis is a colonal disease associated with D816V and other activating C-KIT mutations. J Invest Dermatol 2010; 130:804-815.

13. Yang Y, Létard S, Borge L, Chaix A, Hanssens K, Lopez S, Vita, M, Finetti P, Birnbaum D, Bertucci F, Gomez S, de Sepulveda P, Dubreuil P. Pediatric mastocytosis-associated KIT extracellular domain mutations exhibit different functional and signaling properties compared with KIT-phosphotransferase domain mutations. Blood 2010; 116:1114-1123.

14. Bernd HW, Sotlar K, Lorenzen J, Osieka R, Fabry U, Valent P, Horny HP. Acute myeloid Leukemia (AML) with $\mathrm{t}(8 ; 21)$ associated with "occult" mastocytosis (SM-AHNMD). Report of an unusual case and review of the literature. J Clin Pathol 2004; 57:324-328.

15. Gadage VS, Kadam Amare PS, Galani KS, Mittal N. Systemic mastocytosis with associated acute myeloid leukemia with $\mathrm{t}(8 ; 21)(\mathrm{q} 22 ; \mathrm{q} 22)$. Indian J Pathol Microbiol 2012; 55:409-412.

16. Mahadeo KM, Wolgast L, McMahon C, Cole PD. Systemic mastocytosis in a child with $\mathrm{t}(8 ; 21)$ acute myeloid leukemia. Pediatr Blood Cancer 2011;57:684-687.

17. Frieri M, Linn N, Schweitzer M, Angadi C, Pardanani B. Lymphadenopathic mastocytosis with eosinophilia and biclonal gammopathy. J. Allergy Clin Immunol 1990; 86:126-132.

18. Lindner PS, Pardanani B, Angadi C, Frieri M. Acute nonlymphocytic leukemia in systemic mastocytosis with biclonal gammopathy. J Allergy Clin Immunol 1992; 90:410-412.

19. Kunnath MS, Minnaar G. A case of non-pigment forming cutaneous mastocytosis, masquerading as infantile eczema. Arch Dis Child 2010; 95:A26-A27.

20. McClelland VM, Brookfield DSK. Palpation reveals the diagnosis. Arch Dis Child 2005; 90:1278.

21. Krishnan KR, Ownby DR. A solitary mastocytoma presenting with urticaria and angioedema in a 14-year-old boy. Allergy Asthma Proc 2010; 31:520-523.

22. Hoffmann KM, Moser A, Lohse P, Winkler A, Binder B, Sovinz P, Lackner H, Schwinger W, Benesch M, Urban C. Successful treatment of progressive cutaneous mastocytosis with Imatinib in a 2 year-old boy carrying a somatic KIT mutation. Blood 2008; 112:1655-1657.

23. Moen MD, McKeage K, Plosker GL, Siddiqui MA. Imatinib: a review of its use in chronic myeloid leukaemia. Drugs 2007; 67:299-320.

24. Fernandes EI, de Faria BC, Cartell A, dos Santos BA, Cestari TF. Systemic mastocytosis in childhood: report of 3 cases J Pediatr (Rio J) 2002; 78:176-180.

25. Frieri M, Claus M, Martinez S, Annunziato D, Kosuri S, Lin J. Fever, hemorrhagic bullae, gastritis in pediatric mastocytosis. Ann Allergy 1989; 63:179-183.

26. Lange M, Niedoszytko M, Renke J, Glen J, Nedoszytko B. Clinical aspects of pediatric mastocytosis: a review of 101 cases. J Eur Acad Dermatol Venereol 2013; 1:97-102. 
27. Correia O, Duarte AF, Quirino P, Azevedo R, Delgado L Cutaneous mastocytosis: two pediatric cases treated with topical pimecrolimus. Dermatol Online J 2010; 16:8.

28. Castells M, Metcalfe DD, Escribano L. Diagnosis and treatment of cutaneous mastocytosis in children: practical recommendations. Am J Clin Dermatol 2011; 12:259-270.

29. Synakiewicz A, Stachowicz-Stencel T, Renke J, Lange M, Adamkiewicz-Drożyńska E, Balcerska A. Systemic mastocytosis in children-therapeutic problems. Med Wieku Rozwoj 2013; 17:126-129.
Address correspondence to:

Marianne Frieri, $M D, P h D$

Nassau University Medical Center

Department of Allergy and Immunology 2201 Hempstead Turnpike

East Meadow, NY 11758

E-mail: mfrieri@numc.edu

Received for publication July 14, 2013; accepted after revision October 15, 2013. 